\section{Cobertura da avaliação do estado nutricional no Sistema de Vigilância Alimentar e Nutricional brasileiro: 2008 a 2013}

\author{
Coverage of assessment of nutritional status in \\ the Brazilian Food and Nutritional Surveillance \\ System, 2008-2013
}

\section{Cobertura de la evaluación del estado nutricional en el Sistema de Vigilancia Alimentaria y Nutricional brasileño, de 2008 a 2013}

\section{Resumo}

Este estudo tenciona descrever e analisar a cobertura nacional da avaliação do estado nutricional da população usuária de serviços públicos de saúde registrada no Sistema de Vigilância Alimentar e Nutricional Web (SISVAN), entre 2008 e 2013. Trata-se de um estudo ecológico com dados secundários de sistemas de informação. Os indicadores construídos foram: percentuais de cadastramento e utilização; cobertura total e de dados provenientes do Programa Bolsa Família, corrigidos pel a população usuária do Sistema Único de Saúde (SUS) e descritos segundo as Unidades da Federação (UF), macrorregiões el ou fases do curso da vida. A análise foi realizada por meio de estatística descritiva, regressão linear para a estimativa da variação temporal e correlação de Spearman entre a cobertura total e variáveis sociodemográficas e de saúde. Entre 2008 e 2013, mais de 99\% dos municípios possuíam indivíduo(s) cadastrado(s) e acompanhado(s) no sistema e as maiores frequências e variações totais de cobertura foram alcançadas no Nordeste e Norte. A cobertura nacional variou de 9,78\% a 14,92\%, com tendência estatisticamente significativa de aumento. O maior volume de informações advém de crianças e adolescentes. A participação do Programa Bolsa Família no SISVAN passou de 57,17\% para $85,78 \%$ dos dados. O Índice de Desenvolvimento Humano Municipal e o Produto Interno Bruto per capita das UF mostraram-se inversamente correlacionados à cobertura, e as equipes de saúde da família, positivamente. Os resultados apontam para uma cobertura ainda baixa para um sistema que se pretende universal. Observa-se aumento significativo da cobertura no período e desvelam-se fatores relacionados a esse aumento.

Política Nutricional; Vigilância Nutricional; Cobertura de Serviços Públicos de Saúde
Fabiana Alves do Nascimento 1

Sara Araújo da Silva 2

Patricia Constante Jaime 1

doi: 10.1590/0102-311X00161516

\author{
Correspondência \\ F. A. Nascimento \\ Faculdade de Saúde Pública, Universidade de São Paulo. \\ Av. Dr. Arnaldo 715, São Paulo, SP 01246-904, Brasil. \\ fabiana.nasci1@gmail.com \\ 1 Faculdade de Saúde Pública, Universidade de São Paulo \\ São Paulo, Brasil. \\ 2 Secretaria de Atenção à Saúde, Ministério da Saúde, Brasília, \\ Brasil.
}




\section{Introdução}

O Sistema de Vigilância Alimentar e Nutricional (SISVAN) é um sistema de informação em saúde (SIS), que possibilita o armazenamento de dados e a geração contínua de informações sobre o estado nutricional e o consumo alimentar dos usuários da atenção básica do Sistema Único de Saúde (SUS), a fim de que tais informações propiciem o diagnóstico e o acompanhamento da situação alimentar e nutricional da população brasileira e contribuam para a elaboração de intervenções sobre os fatores de risco associados aos agravos nutricionais e os determinantes sociais da insegurança alimentar e nutricional, tanto no âmbito da assistência individual, quanto no âmbito coletivo. A vigilância nos serviços de saúde, em conjunto com os inquéritos populacionais, as chamadas nutricionais e a produção científica, compõe a vigilância alimentar e nutricional e, consequentemente, as estratégias de vigilância em saúde no país 1,2 .

A vigilância alimentar e nutricional surgiu no Brasil, e em outros países da América, África e Ásia, por uma iniciativa internacional para dar resposta à situação alarmante de fome, desnutrição e carências de micronutrientes, identificada e mapeada em pesquisas pioneiras realizadas nas décadas de 1950 e 1960, tendo Josué de Castro como principal referência no Brasil 3. Compunha, também, um conjunto de políticas sociais compensatórias ao ajuste econômico implementado no contexto das crises econômica e alimentar mundiais, deflagradas no início da década de 1970 4. A vigilância nutricional foi instituída como um dos campos de atuação do SUS por meio da Lei Orgânica da Saúde (Lei no 8.080/1990) 5, em seu artigo 6o. O SISVAN e seus objetivos foram estabelecidos nacionalmente pela Portaria no 1.156/19906.

A primeira grande expansão do sistema ocorreu quando ele foi indicado como pré-requisito para o financiamento e a implantação do Programa de Atendimento aos Desnutridos e às Gestantes em Risco Nutricional - Leite é Saúde, 1994; substituído pelo Programa de Incentivo ao Combate às Carências Nutricionais, 1998; e o último passou a vincular a permanência do município no programa ao envio regular de dados de antropometria 1,7. Nesse período, alguns autores já discutiam a necessidade de ampliação do escopo de indicadores e das fases da vida monitoradas pelo SISVAN 8 .

Cerca de dez anos depois, em um contexto de maior priorização da segurança alimentar e nutricional na agenda política brasileira, foi realizada a unificação de programas de transferência de renda condicionada, para a criação do Programa Bolsa Família, que passou a adotar prioritariamente a renda familiar como critério para a seleção dos beneficiários, e não mais o marcador biológico e de morbidade do risco nutricional/desnutrição 9. Entre as ações de vigilância alimentar e nutricional preconizadas nas condicionalidades de saúde do programa, apenas o público de crianças menores de sete anos e gestantes são citados.

As primeiras experiências de sistemas gerenciais de informações do SISVAN ocorreram no Estado de São Paulo, com o aplicativo "SISVAN/SP” para crianças menores de cinco anos, e no Paraná, com a ação "Pesar e medir no Programa do Lactente”, no começo dos anos 2000 10,11,12,13. A versão "SISVAN Módulo Municipal” foi lançada em 2003 e consistia em um software instalado nos computadores dos serviços de saúde e secretarias municipais e estaduais, sem conexão com a internet, para a digitação das informações coletadas e posterior envio do arquivo digital ao Ministério da Saúde 7,14.

O “SISVAN Módulo Municipal” foi substituído, em 2008, pelo “SISVAN Web”, uma ferramenta disponível via Internet. As principais vantagens de tal mudança, segundo Coutinho et al. 1, foram: a incorporação das curvas de crescimento da Organização Mundial da Saúde (OMS) 2006 e 2007, a possibilidade de registro de indicadores do consumo alimentar em diferentes faixas etárias e a interface de uso mais prático para o usuário do sistema.

Os dados antropométricos e de consumo alimentar dos usuários do SUS são inseridos no sistema pelos trabalhadores da atenção básica, por meio de uma plataforma on-line acessada na unidade ou secretaria de saúde. A administração central desse sistema é realizada pela Coordenação Geral de Alimentação e Nutrição (CGAN) do Ministério da Saúde. Os dados de acompanhamento de estado nutricional inseridos Sistema de Gestão do Programa Bolsa Família (SIGPBF) são migrados para o SISVAN Web semestralmente 2 .

Atualmente, uma das principais barreiras para a ampliação e consolidação dos sistemas é a fragmentação do trabalho ou retrabalho na coleta, digitação e inclusão de dados em diferentes plataformas. Além disso, problemas de falta de estrutura nos municípios 15, ausência de identificador único, 
falhas na padronização da coleta dificultam a transmissão e qualidade dos dados coletados. No bojo da reestruturação do SUS, a estratégia de integração das informações "e-SUS atenção básica", atualmente em fase de implementação, aparece como uma aposta para resolver parte dos problemas.

A viabilização de informações contínuas acerca do estado nutricional da população e da cobertura de programas de alimentação e nutrição, como pesquisas nacionais e sistemas de vigilância, é um desafio para a efetiva implantação de uma agenda global de nutrição. Segundo o Global Nutrition Report 2014 16, metade dos países do mundo não possuía dados necessários para avaliar o progresso das metas globais de 2015 e o investimento na manutenção e atualização de bases de dados nacionais e mundiais sobre nutrição é insuficiente.

Apesar do crescente reconhecimento da vigilância alimentar e nutricional e demanda de monitoramento na atenção básica, poucos estudos 17,18,19,20 avaliaram a cobertura do SISVAN no Brasil, sendo focalizados do ponto de vista do público, território e/ou período avaliado. Por essas razões, o presente estudo tenciona descrever e analisar a cobertura da avaliação do estado nutricional da população usuária de serviços públicos de saúde registrada no SISVAN Web, entre 2008 e 2013, em todo o país.

A contribuição deste estudo para a área da alimentação e nutrição no Brasil é fornecer, de forma inédita, um panorama nacional dos dados do SISVAN, a partir do lançamento de sua versão online, de modo a colaborar com a avaliação da magnitude e distribuição de sua implementação e estimular a ampliação da coleta e uso contínuos das informações.

\section{Materiais e métodos}

Trata-se de um estudo epidemiológico de delineamento ecológico, descritivo, cujo público é a população atendida na atenção básica do SUS entre 2008 e 2013. Os dados do Brasil foram analisados segundo Unidades da Federação (UF), macrorregiões e/ou fases do curso da vida.

Foram utilizados dados secundários provenientes do SISVAN Web (Relatórios Públicos. http:// dabsistemas.saude.gov.br/sistemas/sisvan/relatorios_publicos/relatorios.php, acessado em 02/ Ago/2015); do Departamento de Informática do SUS (DATASUS; http://www2.datasus.gov.br/ DATASUS/index.php?area=02); do Instituto Brasileiro de Geografia e Estatística (IBGE; http:// www.ibge.gov.br/home/); do Departamento de Atenção Básica do Ministério da Saúde (DAB; http:// dab.saude.gov.br/portaldab/historico_cobertura_sf.php); do Cadastro Nacional de Estabelecimentos de Saúde (CNES; http://cnes.datasus.gov.br/); e do Atlas do Desenvolvimento Humano no Brasil 21, produzido pelo Programa das Nações Unidas para o Desenvolvimento (PNUD), Instituto de Pesquisa Econômica Aplicada (IPEA) e Fundação João Pinheiro (FJP). O banco de dados do SISVAN Web foi solicitado à CGAN e os demais foram obtidos por intermédio de relatórios públicos em sítios na Internet.

Os indicadores construídos para averiguar o desempenho do sistema com relação ao acompanhamento do estado nutricional são: percentuais de cadastramento e utilização; cobertura total e de dados provenientes do Programa Bolsa Família, estratificados pelas fases do curso da vida, UF e macrorregiões.

$\mathrm{O}$ percentual de cadastramento se refere à porcentagem de municípios que cadastraram indivíduos no sistema, e foi obtido pela divisão do número de municípios com cadastro no SISVAN Web pelo número total de municípios, multiplicado por 100.

O percentual de utilização se refere à porcentagem de municípios que incluíram, no mínimo, um registro de acompanhamento do estado nutricional no sistema, e foi obtido pela divisão do número de municípios que incluíram registros de acompanhamento do estado nutricional no SISVAN Web pelo número total de municípios, multiplicado por 100.

A cobertura total diz respeito ao percentual de indivíduos acompanhados no SISVAN Web, e foi obtido pelo número de indivíduos com registros de estado nutricional no SISVAN Web dividido pela população usuária do SUS, multiplicado por 100.

A opção pela população usuária do SUS foi tomada a fim de evitar a subestimação da cobertura ao utilizar o denominador população total para a construção do indicador. A população usuária do SUS é aquela que utiliza os serviços públicos e gratuitos e que não dispõe de plano ou seguro privado de 
saúde, assumindo-se ínfimas a parcela possuidora de planos de saúde que realiza acompanhamento nutricional na atenção básica do SUS e aquela que pagaria diretamente os custos do serviço privado. É calculada pela subtração da população residente total (IBGE) pela consumidora de planos privados de saúde (Agência Nacional de Saúde Suplementar - ANS).

A cobertura por fase do curso da vida é estratificada em crianças pré-escolares (0-4 anos), crianças escolares (5-9 anos), adolescentes (10-19 anos), adultos (20-59 anos), idosos (60 anos e mais) e gestantes. Como não havia dados do Censo e da ANS disponíveis para gestantes, essa estimativa foi calculada com base em metodologia adotada previamente 22 , com base no total de gestantes estimadas para o município (número de nascidos vivos do ano anterior $+10 \%$ de perdas resultantes de abortos e subnotificações), multiplicado pelo percentual de população feminina em idade fértil usuária do SUS (população feminina de 10-49 anos subtraída pela população feminina 10-49 anos com planos privados de saúde). Foi utilizado o banco de dados do Sistema de Informação sobre Nascidos Vivos (SINASC; http://sinasc.saude.gov.br/default.asp) para o cálculo da estimativa de gestantes. Esse indicador foi construído para os anos de 2008-2012, pela ausência de dados da população de referência, estratificados por faixa etária, disponíveis para 2013.

O indicador de cobertura proveniente do Programa Bolsa Família trata-se do número de indivíduos com registro de estado nutricional provenientes do acompanhamento das condicionalidades do Programa, dividido pela população usuária do SUS, multiplicado por 100.

As variáveis sociodemográficas e de saúde utilizadas para avaliar a correlação com a cobertura total do acompanhamento do estado nutricional foram: Índice de Desenvolvimento Humano Municipal (IDH-M); Produto Interno Bruto (PIB) per capita; população residente; densidade demográfica; estimativas da população usuária do SUS coberta por agentes comunitários de saúde (ACS) e equipes saúde da família (EqSF); e número de nutricionistas na atenção básica e nas Redes de Atenção à Saúde (RAS). Verificou-se a correlação somente em 2010, devido à disponibilidade das informações.

A análise da cobertura e das estimativas da variação temporal foi realizada por meio de estatística descritiva (média e frequência) e modelo de regressão linear, sendo a cobertura total e o ano as variáveis desfecho e explanatória, respectivamente. O intervalo de confiança foi utilizado para avaliar a significância estatística das variações temporais.

A relação entre os indicadores de cobertura e as variáveis socioeconômicas, demográficas e de saúde foi obtida mediante análise não paramétrica de correlação de Spearman, por conta da distribuição não normal dos dados. Considerou-se nível de significância < 0,05. As análises foram calculadas no software Stata 13.1 (StataCorp LP, College Station, Estados Unidos) e os mapas foram produzidos no programa TabWin para o Windows (DATASUS; http://www2.datasus.gov.br/DATASUS/index. php?area $=060805$ ).

O projeto de pesquisa foi aprovado no Comitê de Ética em Pesquisa da Faculdade de Saúde Pública da Universidade de São Paulo (parecer no 1.040.890), em atendimento à Política Editorial do Ministério da Saúde, aprovada pela Portaria no 884/201123, que disciplina a cessão de dados contidos nas bases nacionais dos SIS geridos pela Secretaria de Atenção à Saúde.

\section{Resultados}

A Tabela 1 apresenta a descrição das UF de acordo com as variáveis socioeconômicas, demográficas e de saúde.

Dos 5.565 municípios brasileiros, 5.564 (99,8\%) possuíam, ao menos, um indivíduo cadastrado e $5.533(99,42 \%)$ apresentavam, no mínimo, um registro de acompanhamento do estado nutricional no sistema em 2010. O mesmo é encontrado nos outros anos do período avaliado (dados não apresentados).

A Tabela 2 mostra a cobertura do acompanhamento do estado nutricional em cada UF e macrorregião, a variação anual média em pontos percentuais e os respectivos intervalos de confiança, entre 2008 e 2013.

Entre 2008 e 2013, as maiores frequências e variações totais de cobertura foram alcançadas nas macrorregiões Nordeste (14,80\%-20,28\%) e Norte (12,54\%-18,55\%). A cobertura média do estado nutricional no país variou de 9,78\%-14,92\%, apresentando tendência estatisticamente significativa de 


\section{Tabela 1}

Caracterização das macrorregiões brasileiras e respectivas Unidades da Federação (UF), segundo variáveis demográficas, socioeconômicas e de saúde no ano de 2010.

\begin{tabular}{|c|c|c|c|c|c|c|c|}
\hline Macrorregiões/UF & $\begin{array}{c}\text { Município } \\
\text { (n) }\end{array}$ & $\begin{array}{c}\text { População } \\
\text { residente } \\
\text { (habitantes) }\end{array}$ & $\begin{array}{c}\text { Densidade } \\
\text { demográfica } \\
\text { (habitantes } / \mathrm{km}^{2} \text { ) }\end{array}$ & $\begin{array}{l}\text { IDH-M } \\
\text { UF }\end{array}$ & $\begin{array}{l}\text { PIB per } \\
\text { capita } \\
\text { (R\$) }\end{array}$ & $\begin{array}{c}\text { População } \\
\text { coberta por } \\
\text { ACS }\end{array}$ & $\begin{array}{c}\text { População } \\
\text { coberta por } \\
\text { EqSF }\end{array}$ \\
\hline Norte & 449 & 15.864 .454 & 4,12 & 0,67 & $12.701,05$ & 12.521 .530 & 7.998 .656 \\
\hline Rondônia & 52 & 1.562 .409 & 6,58 & 0,69 & $15.098,13$ & 1.332 .063 & 872.899 \\
\hline Acre & 22 & 733.559 & 4,47 & 0,66 & $11.567,41$ & 605.682 & 439.928 \\
\hline Amazonas & 62 & 3.483 .985 & 2,23 & 0,67 & $17.173,33$ & 2.342 .848 & 1.682 .314 \\
\hline Roraima & 15 & 450.479 & 2,01 & 0,71 & $14.051,91$ & 279.968 & 280.007 \\
\hline Pará * & 143 & 7.581 .051 & 6,07 & 0,65 & $10.259,20$ & 6.121 .656 & 3.091 .470 \\
\hline Amapá & 16 & 669.526 & 4,69 & 0,71 & $12.361,45$ & 559.156 & 459.166 \\
\hline Tocantins & 139 & 1.383 .445 & 4,98 & 0,70 & $12.461,67$ & 1.280 .157 & 1.172 .872 \\
\hline Nordeste & 1.794 & 53.081 .950 & 34,15 & 0,66 & $9.561,41$ & 46.154 .929 & 38.688 .017 \\
\hline Maranhão & 217 & 6.574 .789 & 19,81 & 0,64 & $6.888,60$ & 5.829 .525 & 5.158 .039 \\
\hline Piauí & 224 & 3.118 .360 & 12,40 & 0,65 & $7.072,80$ & 3.134 .445 & 3.062 .448 \\
\hline Ceará & 184 & 8.452 .381 & 56,76 & 0,68 & $9.216,96$ & 7.038 .189 & 5.867 .195 \\
\hline Rio Grande do Norte & 167 & 3.168 .027 & 59,99 & 0,68 & $10.207,56$ & 2.536 .411 & 2.404 .976 \\
\hline Paraíba & 223 & 3.766 .528 & 66,70 & 0,66 & $8.481,14$ & 3.755 .380 & 3.594 .446 \\
\hline Pernambuco & 185 & 8.796 .448 & 89,63 & 0,67 & $10.821,55$ & 7.476 .302 & 6.023 .396 \\
\hline Alagoas & 102 & 3.120 .494 & 112,33 & 0,63 & $7.874,21$ & 2.420 .454 & 2.256 .794 \\
\hline Sergipe & 75 & 2.068 .017 & 94,35 & 0,67 & $11.572,44$ & 1.924 .642 & 1.741 .156 \\
\hline Bahia & 417 & 14.016 .906 & 24,82 & 0,66 & $11.007,47$ & 12.039 .581 & 8.579 .567 \\
\hline Sudeste & 1.668 & 80.364 .410 & 86,92 & 0,77 & $25.987,86$ & 36.673 .748 & 32.034 .013 \\
\hline Minas Gerais & 853 & 19.597.330 & 33,41 & 0,73 & $17.931,89$ & 14.031 .495 & 13.370 .423 \\
\hline Espírito Santo & 78 & 3.514 .952 & 76,25 & 0,74 & $23.378,74$ & 2.472 .947 & 1.805 .690 \\
\hline Rio de Janeiro & 92 & 15.989 .929 & 365,23 & 0,76 & $25.455,38$ & 6.287 .126 & 5.468 .358 \\
\hline São Paulo & 645 & 41.262 .199 & 166,25 & 0,78 & $30.243,17$ & 13.882 .180 & 11.389 .542 \\
\hline Sul & 1.188 & 27.386 .891 & 48,58 & 0,75 & $22.722,62$ & 15.358 .816 & 13.977 .014 \\
\hline Paraná & 399 & 10.444 .526 & 52,40 & 0,75 & $20.813,98$ & 6.301 .140 & 5.816 .638 \\
\hline Santa Catarina ** & 293 & 6.248 .436 & 65,29 & 0,77 & $24.398,42$ & 4.797 .481 & 4.274 .250 \\
\hline Rio Grande do Sul * & 496 & 10.693 .929 & 39,79 & 0,75 & $23.606,36$ & 4.260 .195 & 3.886 .126 \\
\hline Centro-oeste & 466 & 14.058 .094 & 8,75 & 0,76 & $24.952,88$ & 9.132 .781 & 7.370 .961 \\
\hline Mato Grosso do Sul * & 78 & 2.449 .024 & 6,86 & 0,73 & $17.765,68$ & 2.220 .905 & 1.446 .249 \\
\hline Mato Grosso & 141 & 3.035 .122 & 3,36 & 0,73 & $19.644,09$ & 2.317 .066 & 1.910 .276 \\
\hline Goiás & 246 & 6.003 .788 & 17,65 & 0,74 & $16.251,70$ & 4.046 .835 & 3.600 .436 \\
\hline Distrito Federal & 1 & 2.570 .160 & 444,07 & 0,82 & $58.489,46$ & 547.975 & 414.000 \\
\hline Brasil & 5.565 & 190.755 .799 & 22,43 & 0,73 & $19.766,33$ & 119.841 .804 & 100.068 .661 \\
\hline
\end{tabular}

ACS: agentes comunitários de saúde; EqSF: equipes de saúde da família; IDH-M: Índice de Desenvolvimento Humano Municipal; PIB: Produto Interno Bruto.

Fonte das variáveis: Instituto Brasileiro de Geografia e Estatística (número de municípios, população residente, densidade demográfica, PIB per capita); Atlas de Desenvolvimento Humano (IDH-M); Departamento de Atenção Básica (população coberta por ACS e EqSF); Sistema de Vigilância Alimentar e Nutricional (número de cadastrados e de acompanhados).

* Um município criado posteriormente, em 2013;

** Dois municípios criados posteriormente, em 2013.

aumento (+0,92; IC95\%: 0,14; 1,70), assim como nas macrorregiões Sudeste (+0,82; IC95\%: 0,23; 1,41), Centro-oeste (+0,48; IC95\%: 0,32; 0,64) e Sul (+0,76; IC95\%: 0,40; 1,13).

A Tabela 3 apresenta a cobertura nacional para cada fase do curso da vida, entre 2008 e 2012, bem como a variação anual média em pontos percentuais e os respectivos intervalos de confiança. 
Tabela 2

Cobertura nacional do acompanhamento do estado nutricional no SISVAN Web, com base nas Unidades da Federação (UF) e macrorregiões. Brasil, 2008-2013.

\begin{tabular}{|c|c|c|c|c|c|c|c|c|}
\hline \multirow[t]{2}{*}{ Macrorregiões/UF } & \multicolumn{6}{|c|}{ Cobertura do acompanhamento do estado nutricional (\%) } & \multirow{2}{*}{$\begin{array}{c}\text { Variação anual média (em pontos } \\
\text { percentuais) * }\end{array}$} & \multirow[t]{2}{*}{ IC95\% } \\
\hline & 2008 & 2009 & 2010 & 2011 & 2012 & 2013 & & \\
\hline Norte & 12,54 & 10,61 & 11,96 & 11,90 & 14,47 & 18,55 & 1,19 & $-0,13 ; 2,51$ \\
\hline Rondônia & 9,57 & 8,50 & 8,96 & 8,77 & 11,16 & 10,73 & 0,39 & $-0,22 ; 1,00$ \\
\hline Acre & 11,67 & 10,53 & 10,90 & 11,05 & 16,70 & 19,61 & 1,67 & 0,$05 ; 3,30$ \\
\hline Amazonas & 11,85 & 8,94 & 11,14 & 11,12 & 13,29 & 18,64 & 1,34 & $-0,27 ; 2,95$ \\
\hline Roraima & 9,80 & 12,20 & 14,50 & 13,14 & 14,58 & 19,63 & 1,57 & 0,$48 ; 2,66$ \\
\hline Pará & 13,37 & 11,34 & 12,91 & 12,70 & 15,14 & 20,39 & 1,32 & $-0,21 ; 2,85$ \\
\hline Amapá & 5,29 & 6,41 & 6,16 & 5,91 & 7,44 & 9,36 & 0,66 & 0,$10 ; 1,23$ \\
\hline Tocantins & 17,76 & 14,51 & 14,55 & 15,62 & 19,22 & 21,06 & 0,91 & $-0,64 ; 2,45$ \\
\hline Nordeste & 14,80 & 12,46 & 13,83 & 14,18 & 16,66 & 20,28 & 1,15 & $-0,14 ; 2,44$ \\
\hline Maranhão & 18,51 & 16,03 & 17,07 & 17,40 & 19,60 & 25,59 & 1,33 & $-0,44 ; 3,09$ \\
\hline Piauí & 17,91 & 13,54 & 15,74 & 16,47 & 17,85 & 23,78 & 1,23 & $-0,68 ; 3,14$ \\
\hline Ceará & 19,41 & 14,11 & 15,03 & 15,84 & 19,23 & 22,67 & 0,93 & $-1,13 ; 2,98$ \\
\hline Rio Grande do Norte & 15,06 & 12,66 & 14,16 & 13,06 & 15,65 & 18,39 & 0,70 & $-0,50 ; 1,90$ \\
\hline Paraíba & 16,81 & 15,16 & 16,05 & 16,41 & 18,47 & 22,75 & 1,14 & $-0,13 ; 2,42$ \\
\hline Pernambuco & 8,28 & 9,65 & 10,43 & 10,93 & 12,77 & 15,76 & 1,35 & 0,$78 ; 1,91$ \\
\hline Alagoas & 15,11 & 12,04 & 13,18 & 13,84 & 16,60 & 19,40 & 1,02 & $-0,35 ; 2,39$ \\
\hline Sergipe & 11,61 & 10,76 & 12,41 & 12,73 & 15,92 & 19,07 & 1,52 & 0,$52 ; 2,52$ \\
\hline Bahia & 13,31 & 10,83 & 12,79 & 12,90 & 15,56 & 18,40 & 1,14 & $-0,03 ; 2,30$ \\
\hline Sudeste & 6,71 & 6,40 & 7,21 & 7,47 & 8,57 & 11,07 & 0,82 & 0,$23 ; 1,41$ \\
\hline Minas Gerais & 10,77 & 11,15 & 12,04 & 12,52 & 14,45 & 19,33 & 1,52 & 0,$44 ; 2,59$ \\
\hline Espírito Santo & 12,16 & 9,77 & 10,18 & 10,68 & 12,50 & 13,06 & 0,38 & $-0,48 ; 1,24$ \\
\hline Rio de Janeiro & 3,80 & 3,50 & 4,49 & 4,53 & 5,95 & 8,85 & 0,93 & 0,$23 ; 1,63$ \\
\hline São Paulo & 4,82 & 4,32 & 5,11 & 5,32 & 5,71 & 6,78 & 0,40 & 0,$13 ; 0,68$ \\
\hline Sul & 6,46 & 6,77 & 7,83 & 7,82 & 8,22 & 8,96 & 0,48 & 0,$32 ; 0,64$ \\
\hline Paraná & 7,56 & 8,49 & 9,99 & 10,07 & 10,15 & 10,95 & 0,63 & 0,$31 ; 0,95$ \\
\hline Santa Catarina & 4,93 & 6,03 & 6,08 & 6,35 & 7,17 & 7,35 & 0,45 & 0,$27 ; 0,63$ \\
\hline Rio Grande do Sul & 6,20 & 5,49 & 6,73 & 6,49 & 6,96 & 8,01 & 0,38 & 0,$04 ; 0,72$ \\
\hline Centro-oeste & 6,04 & 6,06 & 6,70 & 7,36 & 8,19 & 9,98 & 0,76 & 0,$40 ; 1,13$ \\
\hline Mato Grosso do Sul & 7,47 & 7,64 & 8,39 & 10,02 & 11,80 & 12,90 & 1,18 & 0,$79 ; 1,57$ \\
\hline Mato Grosso & 7,51 & 6,71 & 8,18 & 8,88 & 9,10 & 11,50 & 0,79 & 0,$26 ; 1,33$ \\
\hline Goiás & 6,48 & 6,92 & 7,49 & 7,66 & 8,14 & 10,17 & 0,63 & 0,$26 ; 1,01$ \\
\hline Distrito Federal & 1,29 & 1,31 & 0,75 & 1,52 & 3,02 & 4,17 & 0,58 & 0,$04 ; 1,12$ \\
\hline Brasil & 9,78 & 8,78 & 9,87 & 10,12 & 11,71 & 14,42 & 0,92 & 0,$14 ; 1,70$ \\
\hline
\end{tabular}

IC95\%: intervalo de 95\% de confiança; SISVAN: Sistema de Vigilância Alimentar e Nutricional.

* Coeficiente da regressão linear.

Com relação à estratificação por fases do curso da vida, o maior volume de informações advém de crianças, gestantes e adolescentes. As gestantes (+2,95; IC95\%: 2,49; 3,40), os pré-escolares (+2,81; IC95\%: 1,74; 3,87), os adultos (+0,64; IC95\%: 0,11; 1,17) e os idosos (+0,15; IC95\%: 0,03; 0,27) apresentaram tendência significativa de aumento da cobertura, e a variação da cobertura das crianças menores de cinco anos passou de $17,69 \%$ a $27,89 \%$ e a das gestantes, de $4,71 \%$ para $16,96 \%$, no período avaliado. $\mathrm{O}$ aumento médio da frequência desses dois grupos foi de quase três pontos percentuais ao ano, destacando-se das demais fases. Apesar do aumento significativo na cobertura da população idosa, ela apresentou médias e variação anual muito baixas (0,41\%-1,17\%) (Tabela 3).

Por macrorregiões e UF, as maiores variações anuais ocorreram no grupo das crianças menores de cinco anos, especialmente no Centro-oeste e Sudeste, com destaque para as UF Mato Grosso do Sul (+6,01; IC95\%: 3,58; 8,45) e Minas Gerais (+5,24; IC95\%: 2,04; 8,45) (dados não apresentados). 
Variação temporal da cobertura do acompanhamento do estado nutricional no SISVAN Web, segundo as fases do curso da vida. Brasil, $2008-2012$.

\begin{tabular}{|c|c|c|c|c|c|c|c|}
\hline \multirow[t]{2}{*}{ Fases do curso da vida } & \multicolumn{5}{|c|}{ Cobertura do estado nutricional (\%) } & \multirow{2}{*}{$\begin{array}{c}\text { Variação anual média (em } \\
\text { pontos percentuais) * }\end{array}$} & \multirow[t]{2}{*}{ IC95\% } \\
\hline & 2008 & 2009 & 2010 & 2011 & 2012 & & \\
\hline$<5$ anos & 17,69 & 20,29 & 29,08 & 27,95 & 27,89 & 2,81 & 1,$74 ; 3,87$ \\
\hline $5-9$ anos & 20,19 & 14,45 & 17,83 & 17,88 & 19,37 & 0,18 & $-0,60 ; 0,96$ \\
\hline 10-19 anos & 11,33 & 8,27 & 8,27 & 8,56 & 11,51 & 0,06 & $-0,46 ; 0,59$ \\
\hline 20-59 anos & 8,74 & 8,37 & 8,93 & 9,32 & 11,48 & 0,64 & 0,$11 ; 1,17$ \\
\hline 60 e mais anos & 0,41 & 1,10 & 0,82 & 1,13 & 1,17 & 0,15 & 0,$03 ; 0,27$ \\
\hline Gestantes ** & 4,71 & 8,45 & 10,57 & 13,43 & 16,96 & 2,95 & 2,$49 ; 3,40$ \\
\hline
\end{tabular}

IC95\%: intervalo de 95\% de confiança;

* Coeficiente da regressão linear;

** Estimativa específica para gestante usuária do SUS: (número de nascidos vivos do ano anterior + 10\% de perdas resultantes de abortos e subnotificações) x percentual de população feminina em idade fértil usuária do SUS.

Entre 2008 e 2013, a participação do Programa Bolsa Família na vigilância do estado nutricional no Brasil passou de 57,17\% para 85,78\% dos dados (Tabela 4), e o salto ocorreu de 2008 para 2009, quando aumentou $24,89 \%$.

A Tabela 5 mostra a Correlação de Spearman da cobertura total do SISVAN Web em relação às variáveis demográficas, socioeconômicas e de saúde nas UF em 2010.

Em 2010, o IDH-M e o PIB per capita das UF mostraram-se inversamente correlacionados à cobertura do acompanhamento do estado nutricional, e as coberturas de EqSF e ACS foram positivamente correlacionadas.

\section{Discussão}

A presença de cadastro e de utilização em mais de 99\% dos municípios brasileiros mostra a vinculação formal ao sistema e a existência, em alguma medida, de alimentação do SISVAN Web com relação aos indicadores de acompanhamento nutricional. Em 2008, 50\% dos municípios brasileiros já incluíam informações do SISVAN Web e 95\% registravam dados antropométricos no SIGPBF 1.

Segundo estudo baseado na população de crianças menores de dois anos usuárias do SUS da Superintendência Regional de Saúde de Belo Horizonte (SRS-BH), o percentual de municípios que apresentaram acompanhamento registrado entre 2008 e 2011 variou de $61,5 \%$ para 69,2\% no SISVAN Web e $94,9 \%$ para $100 \%$ no SIGPBF 18 . Em outro estudo realizado com base na população total do Rio Grande do Sul, em 2010, constatou-se percentual de utilização de 65,3\% e correlação inversa dele com a cobertura Estratégia Saúde da Família (ESF), ao contrário do que se esperava 20.

No que diz respeito à cobertura, o fato de as regiões Nordeste e Norte terem apresentado frequências maiores do que as outras regiões pode ter relação com a priorização, em determinadas políticas de saúde, de localidades em que a pobreza, a fome e a desnutrição infantil estavam mais presentes. Tanto a cobertura ampla da ESF e de beneficiários do Programa Bolsa Família nessas regiões; como a presença de grupos/centros colaboradores e dos projetos embrionários do SISVAN, especialmente no Nordeste, são exemplos de tal construção.

Além disso, a constatação de que as localidades mais ricas e com maiores IDH-M apresentaram correlação inversa com a cobertura indica que a incorporação da prática de vigilância alimentar e nutricional no cotidiano dos serviços de saúde não depende exclusivamente da disponibilidade de recursos financeiros, mas fundamentalmente de outros aspectos, como o comprometimento político com a saúde coletiva e com o SUS por parte de gestores e profissionais responsáveis pelas ações de alimentação e nutrição nos territórios. 
Tabela 4

Contribuição percentual do Programa Bolsa Família no acompanhamento do estado nutricional no SISVAN Web, segundo Unidades da Federação (UF) e macrorregiões. Brasil, 2008-2013.

\begin{tabular}{|c|c|c|c|c|c|c|}
\hline Macrorregiões/UF & $\begin{array}{c}2008 \\
(\%)\end{array}$ & $\begin{array}{c}2009 \\
(\%)\end{array}$ & $\begin{array}{c}2010 \\
(\%)\end{array}$ & $\begin{array}{c}2011 \\
(\%)\end{array}$ & $\begin{array}{c}2012 \\
(\%)\end{array}$ & $\begin{array}{c}2013 \\
(\%)\end{array}$ \\
\hline Norte & 60,85 & 90,00 & 94,57 & 91,34 & 91,15 & 94,39 \\
\hline Rondônia & 62,66 & 91,77 & 95,65 & 93,50 & 92,83 & 96,74 \\
\hline Acre & 67,78 & 98,29 & 97,61 & 91,49 & 82,87 & 95,61 \\
\hline Amazonas & 62,19 & 98,32 & 98,74 & 95,23 & 97,29 & 95,98 \\
\hline Roraima & 61,33 & 98,52 & 98,55 & 99,01 & 96,02 & 99,24 \\
\hline Pará & 59,91 & 86,42 & 92,56 & 90,64 & 92,01 & 94,51 \\
\hline Amapá & 58,22 & 96,42 & 96,75 & 96,11 & 95,83 & 98,72 \\
\hline Tocantins & 59,29 & 84,90 & 92,65 & 83,35 & 78,40 & 86,13 \\
\hline Nordeste & 60,81 & 92,62 & 94,14 & 93,08 & 92,26 & 95,07 \\
\hline Maranhão & 61,26 & 94,45 & 97,19 & 97,24 & 95,97 & 97,50 \\
\hline Piauí & 63,37 & 97,86 & 98,09 & 97,75 & 97,03 & 97,48 \\
\hline Ceará & 60,59 & 96,17 & 97,34 & 96,21 & 93,44 & 97,27 \\
\hline Rio Grande do Norte & 62,33 & 95,02 & 95,90 & 95,25 & 93,61 & 94,24 \\
\hline Paraíba & 59,01 & 84,17 & 86,17 & 84,95 & 86,03 & 89,54 \\
\hline Pernambuco & 58,94 & 90,17 & 93,58 & 91,22 & 89,66 & 93,78 \\
\hline Alagoas & 59,93 & 93,36 & 93,33 & 90,25 & 88,55 & 92,53 \\
\hline Sergipe & 63,86 & 92,19 & 96,14 & 95,45 & 95,60 & 96,33 \\
\hline Bahia & 60,60 & 90,77 & 91,41 & 90,23 & 91,26 & 94,29 \\
\hline Sudeste & 53,43 & 71,67 & 75,48 & 72,96 & 70,48 & 72,45 \\
\hline Minas Gerais & 55,34 & 64,48 & 69,74 & 67,47 & 61,80 & 59,44 \\
\hline Espírito Santo & 49,34 & 72,47 & 75,93 & 72,59 & 71,60 & 80,32 \\
\hline Rio de Janeiro & 54,47 & 92,55 & 89,53 & 87,83 & 87,90 & 91,53 \\
\hline São Paulo & 51,45 & 75,93 & 78,47 & 75,71 & 75,79 & 83,01 \\
\hline Sul & 45,82 & 59,23 & 62,20 & 58,31 & 58,64 & 66,89 \\
\hline Paraná & 48,28 & 60,73 & 61,96 & 58,29 & 57,83 & 66,30 \\
\hline Santa Catarina & 33,27 & 41,06 & 49,59 & 42,43 & 45,05 & 53,47 \\
\hline Rio Grande do Sul & 48,23 & 67,94 & 69,35 & 67,49 & 68,29 & 75,19 \\
\hline Centro-oeste & 53,31 & 73,81 & 81,97 & 75,14 & 77,14 & 84,77 \\
\hline Mato Grosso do Sul & 50,27 & 69,24 & 72,47 & 68,46 & 66,53 & 73,70 \\
\hline Mato Grosso & 46,41 & 74,66 & 82,15 & 72,30 & 80,55 & 86,10 \\
\hline Goiás & 59,78 & 76,91 & 86,67 & 82,90 & 83,17 & 89,09 \\
\hline Distrito Federal & 39,53 & 50,38 & 65,33 & 38,82 & 61,59 & 87,29 \\
\hline Brasil & 57,16 & 82,03 & 85,11 & 82,72 & 82,32 & 85,78 \\
\hline
\end{tabular}

SISVAN: Sistema de Vigilância Alimentar e Nutricional.

Enes et al. 19 apontam algo semelhante sobre a cobertura do SISVAN no Estado de São Paulo. Por dados dos relatórios públicos de 2010 e da população usuária do SUS, verificou-se cobertura baixa $(<10 \%)$ na maioria das regiões administrativas do estado, valor correspondente ao estimado neste trabalho no mesmo ano (5,11\%). As possíveis razões apontadas incluem: a ausência de registros ou regularidade no envio das informações para o DATASUS; pouca apropriação de métodos de planejamento local por parte dos profissionais de saúde; a tradicional centralização e verticalização das políticas sociais no Brasil; a concorrência entre os programas por causa das dificuldades de implementação de um sistema de informação unificado; e a falta de compromisso político por parte de alguns gestores.

No Rio Grande do Sul, Jung et al. 20 descreveram coberturas de acompanhamento do estado nutricional por faixa etária com base nos relatórios públicos e na população total de 496 municípios, 
Correlação de Spearman da cobertura da avaliação do estado nutricional no SISVAN Web em relação às variáveis demográficas, socioeconômicas e de saúde em 2010.

\begin{tabular}{lcc}
\hline Variáveis demográficas, socioeconômicas e de saúde & Correlação de Spearman & Valor de $\mathbf{p}$ \\
\hline Cobertura do estado nutricional no SISVAN Web & & 0,510 \\
População residente (habitantes) & $-0,1327$ & 0,240 \\
Densidade demográfica (habitantes $\left./ \mathrm{km}^{2}\right)$ & $-0,2338$ & $<0,001$ \\
IDH-M UF & $-0,8029$ & $<0,001$ \\
PIB per capita $(\mathrm{R} \$)$ & $-0,8455$ & 0,019 \\
Cobertura de ACS & 0,4481 & 0,002 \\
Cobertura das equipes de saúde da família & 0,5635 & 0,458 \\
Nutricionistas na atenção básica & $-0,15$ & 0,300 \\
Nutricionistas no SUS & $-0,21$ & \\
\hline
\end{tabular}

ACS: agentes comunitários de saúde; ESF: Estratégia Saúde da Família; IDH-M: Índice de Desenvolvimento Humano Municipal; PIB: Produto Interno Bruto; SISVAN: Sistema de Vigilância Alimentar e Nutricional; SUS: Sistema Único de Saúde; UF: Unidades da Federação.

em 2010. As frequências de acompanhamento do estado nutricional foram maiores entre crianças de 0 a 5 anos (10,5\%) e de 5 a 10 anos (5,1\%), e muito baixas entre adolescentes $(1,6 \%)$, adultos $(0,8 \%)$ e idosos (0,9\%). A preponderância do acompanhamento de crianças e a cobertura reduzida entre idosos também foi verificada em outros estudos 1,19 .

Essas características da cobertura estão vinculadas à histórica priorização do componente materno-infantil, considerado biologicamente vulnerável, nas políticas de saúde como um todo e, mais especificamente, nas relacionadas à alimentação e nutrição, desde a Campanha de Merenda Escolar - voltada às crianças - e o Programa Nacional de Alimentação e Nutrição (PRONAN), que definiu crianças, gestantes e nutrizes como público-alvo 24. Como a principal fonte de dados do SISVAN é o Programa Bolsa Família, cujo público prioritário é o mesmo, o acompanhamento do estado nutricional dessa fase da vida é reforçado.

Tal perfil fazia mais sentido no contexto de altas prevalências de desnutrição infantil em todo o país; entretanto é dissonante com o cenário atual de transição demográfica, epidemiológica e nutricional 25,26. Com o aumento acelerado da obesidade e de condições associadas, particularmente nas fases da adolescência e da vida adulta 27, as novas necessidades de atenção nutricional precisam ser identificadas pela vigilância alimentar e nutricional, e para isso, o aumento da cobertura nas demais fases do curso da vida é pré-requisito.

Ainda sobre a participação do Programa Bolsa Família na obtenção de dados para a vigilância alimentar e nutricional, além da marcante participação na definição do público majoritário, é notória a importância do programa como disseminador do uso do SISVAN e impulsionador das ações de vigilância. Uma possível explicação para o salto observado nos dados provenientes do Programa Bolsa Família, de 2008 (57,17\%) para 2009 (82,06\%), seria a transição do SISVAN Módulo Municipal para Web, que ocorreu no primeiro semestre de 2008 18. Os dados das famílias beneficiárias do Programa Bolsa Família eram coletados no SIGPBF e passaram a ser migrados para o SISVAN Web com maior eficiência.

Ao mesmo tempo, essa centralidade do Programa Bolsa Família, e programas anteriores, na definição do público prioritário do SISVAN estimula uma visão limitada do município com relação ao sistema, de forma que a sua identidade sempre esteja vinculada ao público específico desses programas, dificultando a extrapolação para outros cenários.

A correlação positiva entre a cobertura da avaliação do estado nutricional e a cobertura de ACS e EqSF confirma a previsão, já que a ESF é considerada a tática governamental prioritária para expansão, consolidação e qualificação da atenção básica. Programas de atenção primária baseados na família e orientados para a comunidade, como a ESF e os ACS, foram associados à reorientação do processo 
de trabalho no sentido do trabalho em equipe e da interdisciplinaridade, maior custo-efetividade frente a outras propostas assistenciais, melhora da resolutividade e impacto positivo na saúde da população com a redução da mortalidade infantil, morbimortalidade por doenças cardiovasculares e de internações por doenças preveníveis a partir de cuidados primários 28,29,30,31,32,33.

A promoção da alimentação saudável está inserida no rol de atribuições das equipes de saúde, e a inserção de dados no SISVAN foi preconizada como pré-requisito para a manutenção do financiamento do piso da atenção básica ${ }^{34}$. No entanto, a baixa cobertura nacional do SISVAN, que variou de 9,8\% a 14,9\% entre a população usuária do SUS, em comparação com a da ESF, que passou de 49,5\% a 56,4\% entre a população total (http://dab.saude.gov.br/portaldab/historico_cobertura_sf.php), mostra um descompasso no acompanhamento nutricional e uso do sistema pelas equipes.

Alguns estudos 14,18,35 se debruçaram sobre a implantação e implementação do SISVAN e seu papel no ciclo de gestão e produção do cuidado em alimentação e nutrição, bem como as limitações do sistema e da vigilância alimentar e nutricional. Os principais problemas relacionados à operacionalização do sistema e uso das informações coletadas para a elaboração de ações, geralmente, podem ser identificados em um ou mais desses elementos, segundo Camilo et al. 36: estrutura física, capacitação dos profissionais; comunicação e colaboração entre as esferas de governo; interatividade e inteligência do sistema.

Na literatura, a subutilização das informações do SISVAN no Brasil e a concentração do trabalho em vigilância alimentar e nutricional nas primeiras etapas do ciclo também foram relacionadas à implantação do sistema de forma isolada no setor saúde 37 ; sustentação política pouco consistente ${ }^{3,37}$; baixa cobertura geográfica e populacional 3,17,18,20; dados produzidos não acionarem intervenções no governo 3,4; atrelamento do sistema ao recebimento de recursos para programas assistenciais 36; pouca utilização dos dados do SISVAN em outros países 38; insuficiência da exigência formal para a utilização local das informações 39; pouca parceria multissetorial na gestão do sistema 14; descontinuidade no envio dos dados 1,12; baixa qualidade ou falta de confiança neles 3,17; dificuldade em analisá-los e interpretá-los 40; ausência de estudos de validação para aumentar a confiabilidade das informações 41 ; dados não serem representativos da população brasileira 18; insuficiência de nutricionistas e sobrecarga de trabalho dos profissionais na operacionalização do SISVAN; conteúdo de documentos de orientação e capacitações com enfoque na coleta e digitação dos dados, na antropometria e no cuidado com a criança; falta de formação dos profissionais em gestão pública ou não apropriação deste instrumental; a não vinculação formal do SISVAN do município à atenção básica, levando a certa fragilidade na institucionalização 35 , entre outras.

Faz-se necessário apresentar os elementos que podem interferir na interpretação dos resultados.

Os resultados deste estudo não possibilitam inferências em nível individual, uma vez que se trata de um estudo de agregados com análises completamente ecológicas. A respeito de as associações ecológicas serem dependentes do tamanho das unidades de agregação, as análises do presente estudo foram realizadas com base nas UF, menores unidades geográficas disponíveis, a fim de minimizar o problema. O uso de dados secundários se apresenta como uma limitação, já que são provenientes de fontes diversas e, consequentemente, possuem uma variação na confiabilidade das informações, advinda de sub-regitro, erros na coleta, digitação e armazenamento dos dados, entre outros. A obtenção das informações realizada em bases de dados nacionais amplamente utilizadas em estudos epidemiológicos e a análise de consistência criteriosa foram cuidados adotados.

A opção de análise deste estudo pode apresentar-se como uma limitação. Discussões recentes sobre estudos ecológicos mistos apontam o uso de modelagens mais sofisticadas, que pressuponham a ocorrência de autocorrelação espacial e temporal 42 . O tamanho reduzido da série e o caráter exploratório do estudo, sem pretensão de gerar previsões de valores futuros, foram elementos considerados na análise.

Recomenda-se cautela na interpretação do percentual de cadastro e utilização, pois foram incluídos municípios com ao menos um registro, o que pode levar a conclusões equivocadas a respeito do desempenho do sistema porque é possível encontrar localidades com alto percentual de utilização e baixas coberturas. A ausência de algumas informações disponíveis para o cálculo dos indicadores também pode ser mencionada como possível limitação, tanto nas informações sobre as gestantes da população de referência, quanto a ausência de dados estratificados por fases do curso da vida no ano de 2013. O cálculo da estimativa das gestantes foi uma forma de tentar contornar parcialmente esta questão. 
Em suma, para que o SISVAN possa se reafirmar como uma fonte de informações que subsidia a agenda da alimentação e nutrição nos municípios, nos estados e no país, alguns problemas precisam ser enfrentados. Investimentos em equipamentos, materiais, estrutura das unidades, dinamização dos sistemas informatizados, capacitações em todas as fases do ciclo de gestão e produção do cuidado, entre outros, são fundamentais para o estabelecimento e sustentação de um grau satisfatório de cobertura do sistema. Todavia, os maiores desafios talvez estejam na superação dos entraves políticos (descontinuidade das ações; desencontros entre os interesses da gestão municipal, estadual ou federal, entre outros) e administrativos para garantir a periodicidade do provimento de todos esses recursos e na valorização do SISVAN, perante usuários, profissionais e gestores, enquanto um sistema de informação em saúde necessário para qualificar a gestão da alimentação e nutrição no Brasil.

\section{Conclusão}

Este trabalho complementa as informações sobre a variação da cobertura do SISVAN Web em todo o Brasil.

Os resultados apontam para uma cobertura ainda baixa para um sistema de vigilância alimentar e nutricional que se pretende universal. Por outro lado, verifica-se aumento significativo da cobertura no período e fatores relacionados a esse aumento (IDH-M UF, PIB per capita, coberturas de ACS e EqSF). Pelas estratificações conduzidas e pelas comparações com outros estudos, desvelam-se contextos em que recursos precisam ser mobilizados para melhoria da vigilância alimentar e nutricional. A participação do Programa Bolsa Família como fonte de dados para a vigilância alimentar e nutricional é muito relevante, refletindo tanto na distribuição geográfica do acompanhamento, quanto nas características da população monitorada.

Reconhecendo a centralidade da perspectiva materno-infantil nas ações de alimentação e nutrição, vale ressaltar que o acompanhamento do estado nutricional segue uma tendência de mudança, ainda que lenta, em direção ao cuidado que vise a todas as fases do curso da vida.

\section{Colaboradores}

F. A. Nascimento e P. C. Jaime participaram da concepção e projeto do estudo, análise e interpretação dos dados; redação do artigo e revisão crítica relevante do conteúdo intelectual; aprovação final da versão a ser publicada; e é responsável por todos os aspectos do trabalho. S. A. Silva participou da concepção e projeto do estudo; revisão crítica relevante do conteúdo intelectual; aprovação final da versão a ser publicada; e são responsáveis por todos os aspectos do trabalho na garantia da exatidão e integridade de qualquer parte da obra.

\section{Agradecimentos}

À Maria Laura da Costa Louzada pelo apoio nas análises estatísticas. À Capes pelo financiamento da bolsa de mestrado. 


\section{Referências}

1. Coutinho JG, Cardoso AJC, Toral N, Silva ACF, Ubarana JA, Aquino KKNC, et al. A organização da Vigilância Alimentar e Nutricional no Sistema Único de Saúde: histórico e desafios atuais. Rev Bras Epidemiol 2009; 12:688-699.

2. Departamento de Atenção Básica, Secretaria de Atenção à Saúde, Ministério da Saúde. Marco de referência da vigilância alimentar e nutricional na atenção. Brasília: Ministério da Saúde; 2015.

3. Batista-Filho M, Rissin A. Vigilância alimentar e nutricional: antecedentes, objetivos e modalidades. A VAN no Brasil. Cad Saúde Pública 1993; 9 Suppl 1:99-105.

4. Castro IRR. Vigilância alimentar e nutricional: limitações e interfaces com a rede de saúde. Rio de Janeiro: Editora Fiocruz; 1995.

5. Presidência da República. Lei no 8.080, de 19 de setembro de 1990, dispõe sobre as condições para a promoção, proteção e recuperação da saúde, a organização e o funcionamento dos serviços correspondentes e dá outras providências. Diário Oficial da República Federativa do Brasil 1990; 20 set.

6. Ministério da Saúde. Portaria no 1.156, de 31 de agosto de 1990, institui o Sistema de Vigilância Alimentar e Nutricional - SISVAN. Diário Oficial da República Federativa do Brasil 1990; 5 set.

7. Departamento de Atenção Básica, Secretaria de Atenção à Saúde, Ministério da Saúde. Indicadores de vigilância alimentar e nutricional. Brasília: Ministério da Saúde; 2009.

8. Castro IRR. Boletim Nacional do SISVAN. Brasília: Instituto Nacional de Alimentação e Nutrição; 1996.

9. Trevisani JJD, Burlandy L, Jaime PC. Fluxos decisórios na formulação das condicionalidades de saúde do programa bolsa família. Saúde Soc 2012; 21:492-509.

10. Cortez ECL, Figueiredo, EM, Brandão NF. Desenvolvimento de um sistema e informações gerenciais para o sistema de vigilância alimentar e nutricional - SISVAN no estado de São Paulo. Boletim do Instituto de Saúde 2002; 26:9-11.

11. Lei DLM, Chavez SP, Saldiva SRDM, Stefanini MLR. O sistema de vigilância alimentar e nutricional. Boletim do Instituto de Saúde 2002; $26: 4-8$.
12. Venâncio SI, Levy RB, Saldiva SRDM, Mondini L, Stefanini MLR. Sistema de Vigilância Alimentar e Nutricional no Estado de São Paulo, Brasil: experiência na implementação e avaliação do estado nutricional de crianças. Rev Bras Saúde Matern Infant 2007; 7:213-20.

13. Coordenação de Vigilância Nutricional, Secretaria de Estado da Saúde. Vigilância nutricional nos municípios paranaenses. http:// www.saude.pr.gov.br/modules/conteudo/con teudo.php? conteudo=675 (acessado em 09/ Jun/2016).

14. Fagundes-Romeiro AA. Avaliação da implantação do Sistema de Vigilância Alimentar e Nutricional - SISVAN, no Brasil [Dissertação de Mestrado]. Brasília: Faculdade de Ciências da Saúde, Universidade de Brasília; 2006.

15. Morais RM, Gomes EJ, Costa AL. Os sistemas de informação do SUS: uma perspectiva histórica e as políticas de informação e informática. Revista Nucleus 2014; 11:287-304.

16. International Food Policy Research Institute. Global Nutrition Report 2014: actions and accountability to accelerate the world's progress on nutrition. http://globalnutritionre port.org/2014/11/13/global-nutrition-re port-2014/ (acessado em 18/Jan/2015).

17. Damé PKV, Pedroso MRO, Marinho CL, Gonçalves VM, Duncan BB, Fisher PD, et al. Sistema de Vigilância Alimentar e Nutricional (SISVAN) em crianças do Rio Grande do Sul, Brasil: cobertura, estado nutricional e confiabilidade dos dados. Cad Saúde Pública 2011; 27:2155-65.

18. Ferreira CS, Cherchiglia ML, César CC. O Sistema de Vigilância Alimentar e Nutricional como instrumento de monitoramento da Estratégia Nacional para Alimentação Complementar Saudável. Rev Bras Saúde Matern Infant 2013; 13:167-77.

19. Enes CC, Loiola H, Oliveira MRM. Cobertura populacional do Sistema de Vigilância Alimentar e Nutricional no Estado de São Paulo, Brasil. Ciênc Saúde Coletiva 2014; 19:1543-51.

20. Jung NM, Bairros FS, Neutzling MB. Utilização e cobertura do Sistema de Vigilância Alimentar e Nutricional no Estado do Rio Grande do Sul, Brasil. Ciênc Saúde Coletiva 2014; 19:1379-88. 
21. Programa das Nações Unidas para o Desenvolvimento; Instituto de Pesquisa Econômica Aplicada; Fundação João Pinheiro. Atlas do desenvolvimento humano do Brasil. http:// www.atlasbrasil.org.br/2013/ (acessado em 02/Ago/2015).

22. Departamento de Atenção Básica, Secretaria de Atenção à Saúde, Ministério da Saúde. Programa Nacional de Melhoria do Acesso e da Qualidade da Atenção Básica (PMAQ): manual instrutivo - anexo: ficha de qualificação dos indicadores. Brasília: Ministério da Saúde; 2012.

23. Ministério da Saúde. Portaria no 884, de 13 de dezembro de 2011, estabelece o fluxo para solicitação de cessão de dados dos bancos nacionais dos Sistemas de Informação. Diário Oficial da União 2011; 14 dez.

24. Silva AC. De Vargas a Itamar: políticas e programas de alimentação e nutrição. Estud Av 1995; 9:87-107.

25. Mendes EV. O cuidado das condições crônicas na atenção primária à saúde: o imperativo da consolidação da estratégia da saúde da família. Brasília: Organização Pan-Americana da Saúde; 2012.

26. Jaime PC, Santos LMP. Transição nutricional e a organização do cuidado em alimentação e nutrição na Atenção Básica em saúde. Divulg Saúde Debate 2014; 51:77-85.

27. Instituto Brasileiro de Geografia e Estatística. Pesquisa de orçamentos familiares 2008-2009: antropometria e estado nutricional de crianças, adolescentes e adultos no Brasil. Rio de Janeiro: Instituto Brasileiro de Geografia e Estatística; 2010.

28. Aquino R, Oliveira NF, Barreto ML. Impact of the family health program on infant mortality in brazilian municipalities. Am J Public Health 2009; 99:87-93.

29. Giovanella L, Mendonça MHM, Almeida PF, Escorel S, Senna MCM, Fausto MCR, et al. Saúde da família: limites e possibilidades para uma abordagem integral de atenção primária à saúde no Brasil. Ciênc Saúde Coletiva 2009; 14:783-94.

30. Guanais F, Macinko J. Primary care and avoidable hospitalizations: evidence from Brazil. J Ambul Care Manage 2009; 32:115-22.

31. Secretaria de Atenção à Saúde, Ministério da Saúde. Política nacional de atenção básica. Brasília: Ministério da Saúde; 2011.
32. Macinko J, Oliveira VB, Turci MA, Guanais FC, Bonolo PF, Lima-Costa MF. The influence of primary care and hospital supply on ambulatory care: sensitive hospitalizations among adults in Brazil, 1999-2007. Am J Public Health 2011; 101:1963-70.

33. Rasella D, Harhay MO, Pamponet ML, Aquino $\mathrm{R}$, Barreto ML. Impact of primary health care on mortality from heart and cerebrovascular diseases in Brazil: a nationwide analysis of longitudinal data. BMJ 2014; 349:g4014.

34. Secretaria de Atenção à Saúde, Ministério da Saúde. Política Nacional de Atenção Básica. Brasília: Ministério da Saúde; 2006.

35. Rolim MD, Lima SML, Barros DC, Andrade CLT. Avaliação do SISVAN na gestão de ações de alimentação e nutrição em Minas Gerais, Brasil. Ciênc Saúde Coletiva 2015; 20:235969.

36. Camilo AMB, Camilo GB, Toledo GC, Camilo Júnior RD, Toledo CT. Vigilância nutricional no Brasil: criação e implementação do SISVAN. Rev APS 2011; 14:224-8.

37. Arruda BKG. Sistema de vigilância alimentar e nutricional. Frustrações, desafios e perspectivas. Cad Saúde Pública 1992; 8:96-101.

38. Acosta SJ. Errores en que se puede incurrir en los sistemas de vigilancia alimentaria y nutricional. Rev Cubana Aliment Nutr 2001; 15:6873.

39. Santana LAA, Santos SAC. Sistema de vigilância alimentar e nutricional na implementação do programa Leite é Saúde: avaliação em municípios baianos. Rev Nutr 2004; 17:283-90.

40. Batista-Filho M, Rissin A. A transição nutricional no Brasil: tendências regionais e temporais. Cad Saúde Pública 2003; 19 Suppl 1:S181-91.

41. Leal MC, Bittencourt SA. Informações nutricionais: o que se tem no país? Cad Saúde Pública 1997; 13:551-5.

42. Aquino R, Gouveia N, Teixeira MG, Costa MC, Barreto ML. Estudos ecológicos (desenho de dados agregados). In: Almeida Filho N, Barreto ML, organizadores. Epidemiologia \& saúde: fundamentos, métodos e aplicações. Rio de Janeiro: Editora Guanabara Koogan; 2012. p. 175-85. 


\section{Abstract}

This study aims to describe and analyze nationwide coverage of the assessment of nutritional status in the user population of public health services recorded in Brazil's Online Food and Nutritional Surveillance System (SISVAN) from 2008 to 2013. This was an ecological study of secondary data from information systems. The indicators were: percentages of registration in and utilization of the system; total coverage and coverage of data from the Bolsa Familia Program, corrected according to the user population of the Brazilian Unified National Health System (SUS) and described according to the states of Brazil and Federal District, major geographic regions, and/or life cycle phases. The analysis used descriptive statistics, linear regression for estimation of temporal variation, and Spearman's correlation between total coverage and social, demographic, and health variables. From 2008 to 2013, more than 99\% of Brazil's municipalities (counties) had individuals enrolled and monitored in the system, and the highest frequencies and total variations in coverage appeared in the Northeast and North. Nationwide coverage ranged from $9.78 \%$ to $14.92 \%$, with a statistically significant upward trend. The largest volume of data was for children and adolescents. The Bolsa Família Program's share of data in SISVAN increased from $57.17 \%$ to $85.78 \%$. The municipal human development index and per capita gross domestic product in the states were inversely correlated with coverage, and family health teams were directly correlated. The results indicate persistently low coverage for a system that is intended to be universal. The study showed a significant increase in coverage during the period analyzed and revealed factors related to this increase.

Nutrition Policy; Nutritional Surveillance; State Health Care Coverage

\section{Resumen}

Este estudio pretende describir y analizar la cobertura nacional de la evaluación del estado nutricional de la población usuaria de servicios públicos de salud, registrada en el Sistema de Vigilancia Alimentaria y Nutricional Web (SISVAN), entre 2008 y 2013. Se trata de un estudio ecológico con datos secundarios de sistemas de información. Los indicadores construidos fueron: porcentajes de registro y utilización; cobertura total y de datos, provenientes del Programa Beca Familia, corregidos por la población usuaria del Sistema Único de Salud (SUS) y descritos según las Unidades de la Federación (UF), macrorregiones y/o fases de la trayectoria de vida. El análisis se realizó mediante estadística descriptiva, regresión lineal para la estimativa de la variación temporal y correlación de Spearman entre la cobertura total y variables sociodemográficas y de salud. Entre 2008 y 2013, más de un 99\% de los municipios contaban con individuo(s) registrados(s) y acompañado(s) en el sistema y las mayores frecuencias y variaciones totales de cobertura se alcanzaron en el Nordeste y Norte. La cobertura nacional varió de 9,78\% a $14,92 \%$, con una tendencia estadísticamente significativa de aumento. El mayor volumen de información proviene de niños y adolescentes. La participación del Programa Beca Familia en el SISVAN pasó de $57,17 \%$ a un $85,78 \%$ de los datos. El Índice de Desarrollo Humano Municipal y el Producto Interno Bruto per cápita de las UF se mostraron inversamente correlacionados a la cobertura, y a los equipos de salud de la familia, positivamente. Los resultados indican una cobertura todavía baja para un sistema que pretende ser universal. Se observa un aumento significativo de la cobertura en el periodo y se desvelan factores relacionados con este aumento.

Política Nutricional; Vigilancia Nutricional; Cobertura de Servicios Públicos de Salud
Recebido em 19/Set/2016

Versão final reapresentada em 10/Mar/2017 Aprovado em 22/Mar/2017 